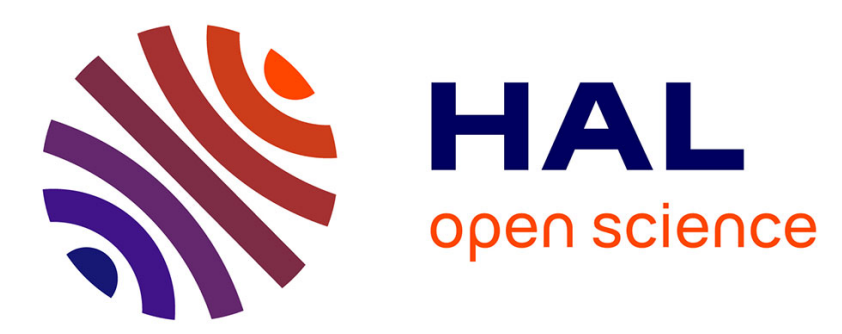

\title{
A variational formulation of the coupled thermo-mechanical boundary-value problem for general dissipative solids
}

Qiang Yang, Laurent Stainier, Michael Ortiz

\section{- To cite this version:}

Qiang Yang, Laurent Stainier, Michael Ortiz. A variational formulation of the coupled thermomechanical boundary-value problem for general dissipative solids. Journal of the Mechanics and Physics of Solids, 2006, 54 (2), pp.401-424. 10.1016/j.jmps.2005.08.010 . hal-01004967

\section{HAL Id: hal-01004967 \\ https://hal.science/hal-01004967}

Submitted on 2 Nov 2021

HAL is a multi-disciplinary open access archive for the deposit and dissemination of scientific research documents, whether they are published or not. The documents may come from teaching and research institutions in France or abroad, or from public or private research centers.
L'archive ouverte pluridisciplinaire HAL, est destinée au dépôt et à la diffusion de documents scientifiques de niveau recherche, publiés ou non, émanant des établissements d'enseignement et de recherche français ou étrangers, des laboratoires publics ou privés.

\section{(ㄷ)(1) $\$$}

Distributed under a Creative Commons Attribution - NonCommerciall 4.0 International 


\title{
A variational formulation of the coupled thermo-mechanical boundary-value problem for general dissipative solids
}

\author{
Q. Yang ${ }^{\mathrm{a}}$, L. Stainier ${ }^{\mathrm{b}}$, M. Ortiz ${ }^{\mathrm{a}, *}$ \\ ${ }^{\mathrm{a}}$ Division of Engineering and Applied Science, California Institute of Technology, Pasadena, CA 91125-5000, USA \\ ${ }^{\mathrm{b}}$ Department of Aerospace, Mechanics and Materials, Université de Liège, B-4000 Liège, Belgium
}

\begin{abstract}
A variational formulation of the coupled thermo-mechanical boundary-value problem for general dissipative solids is presented. The coupled thermo-mechanical boundary-value problem under consideration consists of the equilibrium problem for a deformable, inelastic and dissipative solid with the heat conduction problem appended in addition. The variational formulation allows for general dissipative solids, including finite elastic and plastic deformations, non-Newtonian viscosity, rate sensitivity, arbitrary flow and hardening rules, as well as heat conduction. We show that a joint potential function exists such that both the conservation of energy and the balance of linear momentum equations follow as Euler-Lagrange equations. The identification of the joint potential requires a careful distinction between equilibrium and external temperatures, which are equal at equilibrium. The variational framework predicts the fraction of dissipated energy that is converted to heat. A comparison of this prediction and experimental data suggests that $\alpha$-titanium and Al2024-T conform to the variational framework.
\end{abstract}

Keywords: Variational principles; Irreversible processes; Coupled problems; Viscoplasticity; Heat conduction

\footnotetext{
${ }^{*}$ Corresponding author. Tel.: + 16263954530 ; fax: + 16263040175 .

E-mail address: ortiz@aero.caltech.edu (M. Ortiz).
} 


\section{Introduction}

This paper is concerned with the formulation of variational principles characterizing the solutions of the coupled thermo-mechanical problem for general dissipative solids, here understood as the equilibrium problem of an inelastic deformable solid to which the heat conduction problem is appended in addition. Problems of this nature arise in a variety of important fields of application, including: metal forming, machining, casting and other manufacturing processes; high-velocity impact such as ballistic penetration; and others. By a general dissipative solid we understand a deformable solid, possibly undergoing large deformations, possessing viscosity, internal processes and capable of conducting heat. By a variational characterization of the thermo-mechanical problem we specifically mean the identification of a functional whose stationary points are solutions of the problem. Once this functional is known, the stable solutions of the problem may be identified with certain extrema of the functional, should any exist.

Following the pioneering work of Biot (1956, 1958), the variational form of the coupled thermoelastic and thermoviscoelastic problems has been extensively investigated (Herrmann, 1963; Ben-Amoz, 1965; Oden and Reddy, 1976; Molinari and Ortiz, 1987; Batra, 1989). In addition, at present there are well-developed variational principles for the equilibrium problem of general dissipative solids in the absence of heat conduction (Han et al., 1997a, b; Ortiz and Stainier, 1999). By contrast, the case of thermo-mechanical coupling in dissipative materials has received comparatively less attention (cf., Simo and Miehe, 1992; Armero and Simo, 1992, 1993, for notable exceptions).

When the equilibrium and heat conduction problems for general dissipative solids are combined, the resulting coupled problem lacks an obvious variational structure. This lack of variational structure reveals itself upon linearization of the coupled problem, which results in a non-symmetric operator. This essential difficulty accounts for the lack of variational formulations of the coupled thermo-mechanical problem for general dissipative solids. However, in this paper we show that an integrating factor exists which delivers the sought variational structure. The ability to identify such an integrating factor hinges critically on a careful distinction between two types of temperature: an equilibrium temperature, which follows as a state variable; and an external temperature, which equals the equilibrium temperature at equilibrium. Specifically, we investigate integrating factors that follow from a temperature-dependent rescaling of time in all rate processes. We show that, within this class, the integrating factor is unique. Once the requisite integrating factor is identified, potentials can be determined whose critical points are the solutions of the coupled thermo-mechanical problem in both its rate and incremental forms.

The ability to recast the coupled thermo-mechanical problem in variational form has a number of consequences and some beneficial effects. For instance, the variational framework opens the way for the application of the tools of calculus of variations to the analysis of the solutions of the problem. In particular, conditions for the existence and uniqueness of solutions follow from the direct method of the calculus of variations (cf., e.g., Dal Maso, 1993). In addition, localization phenomena such as shear bands can be effectively studied within the framework of free-discontinuity problems (cf., e.g., Braides, 1998; Ambrosio, 2000). A variational statement of the problem also facilitates the formulation of numerical approximations, e.g., by means of Galerkin or Rayleigh-Ritz methods. In addition, in its time-discretized form the variational framework leads to the 
formulation of robust and efficient state-update algorithms in computational thermoviscoplasticity (Ortiz and Stainier, 1999).

However, perhaps the most far-reaching consequence of the existence of a variational framework is that it places constraints on the constitutive behavior of solids. In particular, if the thermo-mechanical behavior of a solid is variational then the rate of conversion of dissipated work to heat cannot be specified arbitrarily. Instead, it is predicted by the theory. Conversely, the predicted rate of heating can be used as a means of testing if the thermo-mechanical behavior of a solid is variational. We perform this test for two different materials: $\alpha$-titanium and A12024-T3. In particular, we calculate temperature histories under the conditions of the Kolsky (split-Hopkinson) pressure bar experiment and compare the predictions with the measurements of Hodowany et al. (2000). In both cases the comparison is excellent, which suggests that the thermomechanical behavior of $\alpha$-titanium and A12024-T3 does indeed conform to the variational framework.

\section{General framework}

We shall be concerned with the motions and thermodynamic processes undergone by a continuous body of reference configuration $B \subset \mathbb{R}^{3}$. The motions of the body are described by a time-dependent deformation mapping $\varphi: B \times[a, b] \rightarrow \mathbb{R}^{3}$, where $[a, b]$ is the time interval elapsed during the motion. The motions of the body obey conservation of mass

$$
\frac{\mathrm{d}}{\mathrm{d} t} \int_{U} R \mathrm{~d} V=0
$$

conservation of linear momentum

$$
\frac{\mathrm{d}}{\mathrm{d} t} \int_{U} R \boldsymbol{V} \mathrm{d} V=\int_{U} R \boldsymbol{B} \mathrm{d} V+\int_{\partial U} \boldsymbol{P} \boldsymbol{N} \mathrm{d} S
$$

conservation of angular momentum

$$
\frac{\mathrm{d}}{\mathrm{d} t} \int_{U} \boldsymbol{\varphi} \times(R \boldsymbol{V}) \mathrm{d} V=\int_{U} \boldsymbol{\varphi} \times(R \boldsymbol{B}) \mathrm{d} V+\int_{\partial U} \boldsymbol{\varphi} \times(\boldsymbol{P N}) \mathrm{d} S,
$$

the first law of thermodynamics

$$
\begin{aligned}
& \frac{\mathrm{d}}{\mathrm{d} t} \int_{U} R E \mathrm{~d} V+\frac{\mathrm{d}}{\mathrm{d} t} \int_{U} \frac{1}{2} R|\boldsymbol{V}|^{2} \mathrm{~d} V \\
& \quad=\int_{U} R \boldsymbol{B} \cdot \boldsymbol{V} \mathrm{d} V+\int_{\partial U}(\boldsymbol{P N}) \cdot \boldsymbol{V} \mathrm{d} S+\int_{U} R Q \mathrm{~d} V-\int_{\partial U} \boldsymbol{H} \cdot \boldsymbol{N} \mathrm{d} S,
\end{aligned}
$$

and the second law of thermodynamics

$$
\frac{\mathrm{d}}{\mathrm{d} t} \int_{U} N \mathrm{~d} V-\int_{U} \frac{R Q}{T} \mathrm{~d} V+\int_{\partial U} \frac{\boldsymbol{H} \cdot \boldsymbol{N}}{T} \mathrm{~d} S \geqslant 0,
$$

where $U \subset B$ is an arbitrary subbody; $R$ is the mass density per unit undeformed volume; $\boldsymbol{V}=\dot{\boldsymbol{\varphi}}$ is the material velocity; $\boldsymbol{B}$ is the body force density per unit mass; $\boldsymbol{N}$ is the unit outward normal; $\boldsymbol{P}$ is the first Piola-Kirchhoff stress tensor; $E$ is the internal energy per unit undeformed volume; $N$ is the entropy per unit undeformed volume; $T$ is the absolute temperature; $Q$ is the distributed heat source per unit mass; and $\boldsymbol{H}$ is the outward heat flux. 
Alternatively, these conservation laws can be expressed in local form as

$$
\begin{aligned}
& \dot{R}=0, \\
& R \dot{\boldsymbol{V}}=\operatorname{Div} \boldsymbol{P}+R \boldsymbol{B}, \\
& \boldsymbol{P} \boldsymbol{F}^{\mathrm{T}}=\boldsymbol{F P}^{\mathrm{T}}, \\
& \dot{E}=\boldsymbol{P} \cdot \dot{\boldsymbol{F}}+R Q-\operatorname{Div} \boldsymbol{H}, \\
& \dot{\Gamma} \equiv \dot{N}-\frac{R Q}{T}+\operatorname{Div} \frac{\boldsymbol{H}}{T} \geqslant 0,
\end{aligned}
$$

where $\boldsymbol{F}=\operatorname{Grad} \boldsymbol{\varphi}$ is the deformation gradient and $\dot{\Gamma}$ is the internal entropy production rate per unit undeformed volume.

In addition, we suppose that the local thermodynamic state of an infinitesimal material neighborhood is defined by: the local deformation gradient $\boldsymbol{F} \in G L_{+}(3, \mathbb{R}) \equiv$ the Lie group of invertible and orientation-preserving linear transformations in $\mathbb{R}^{3}$; the local entropy density per unit undeformed volume $N \in \mathbb{R}$; and a collection $\boldsymbol{Z} \in M$ of additional or internal variables. The set $M$ in which $\boldsymbol{Z}$ takes values varies depending on the material class and cannot be specified universally for all solids. Depending on the nature of the internal variables, $M$ may be: a vector space; a manifold, e.g., if the internal processes are subject to holonomic constraints; or a Lie group, e.g., if the internal variables are naturally composed by multiplication. In addition, the attainable internal variable rates may be subject to nonholonomic constraints of the type

$$
L(Z) \dot{Z}=\mathbf{0} .
$$

We shall assume that the internal energy density and the absolute temperature are functions of the local state, i.e.,

$$
\begin{aligned}
& E=E(\boldsymbol{F}, N, \boldsymbol{Z}), \\
& T=T(\boldsymbol{F}, N, \boldsymbol{Z}) .
\end{aligned}
$$

The equilibrium stresses and the thermodynamic driving forces conjugate to the internal variables are, by definition,

$$
\begin{aligned}
\boldsymbol{P}^{\mathrm{e}} & \equiv \partial_{\boldsymbol{F}} E(\boldsymbol{F}, N, \boldsymbol{Z}), \\
\boldsymbol{Y} & \equiv-\partial_{\boldsymbol{Z}} E(\boldsymbol{F}, N, \boldsymbol{Z}) .
\end{aligned}
$$

The viscous or non-equilibrium stress is then

$$
\boldsymbol{P}^{\mathrm{v}} \equiv \boldsymbol{P}-\boldsymbol{P}^{\mathrm{e}}
$$

A theorem of Coleman and Noll (1963) then shows that (2.8b) is necessarily of the form

$$
T=\partial_{N} E(\boldsymbol{F}, N, \boldsymbol{Z}),
$$

and that all processes must comply with the dissipation inequality

$$
T \dot{\Gamma}=\boldsymbol{Y} \cdot \dot{\boldsymbol{Z}}+\boldsymbol{P}^{\mathrm{v}} \cdot \dot{\boldsymbol{F}}-\frac{1}{T} \boldsymbol{H} \cdot \operatorname{Grad} T \geqslant 0 .
$$


Expanding $\dot{E}$ in (2.6d) and using the equilibrium relations (2.9a), (2.9b), (2.11) and (2.10) the energy equation takes the entropy form

$$
T \dot{N}=\boldsymbol{P}^{\mathrm{v}} \cdot \dot{\boldsymbol{F}}+\boldsymbol{Y} \cdot \dot{\boldsymbol{Z}}-\operatorname{Div} \boldsymbol{H}+R Q .
$$

Alternatively, we may introduce the Helmholtz free energy by applying the Legendre transformation

$$
A(\boldsymbol{F}, T, \boldsymbol{Z})=\inf _{N}\{E(\boldsymbol{F}, N, \boldsymbol{Z})-T N\},
$$

in terms of which the equilibrium relations take the form

$$
\begin{aligned}
N & =-\frac{\partial A}{\partial T}(\boldsymbol{F}, T, \boldsymbol{Z}), \\
\boldsymbol{P}^{\mathrm{e}} & =\frac{\partial A}{\partial \boldsymbol{F}}(\boldsymbol{F}, T, \boldsymbol{Z}), \\
\boldsymbol{Y} & =-\frac{\partial A}{\partial \boldsymbol{Z}}(\boldsymbol{F}, T, \boldsymbol{Z}) .
\end{aligned}
$$

Example 2.1 (State functions for thermoelastic-viscoplastic materials). A theory of thermoelastic-viscoplastic materials may be based on a multiplicative decomposition of the deformation gradient $\boldsymbol{F}$ of the form (Lee, 1969)

$$
\boldsymbol{F}=\boldsymbol{F}^{\mathrm{e}} \boldsymbol{F}^{\mathrm{p}}
$$

into an elastic part $\boldsymbol{F}^{\mathrm{e}}$ and a plastic part $\boldsymbol{F}^{\mathrm{p}}$. For metals, the elasticity and the specific heat of the material may be assumed to be ostensibly independent of the internal processes. This leads to a free energy of the form (Ortiz and Stainier, 1999):

$$
A\left(\boldsymbol{F}^{\mathrm{e}}, T, \boldsymbol{F}^{\mathrm{p}}, \boldsymbol{Z}\right)=W^{\mathrm{e}}\left(\boldsymbol{F}^{\mathrm{e}}, T\right)+W^{\mathrm{p}}\left(T, \boldsymbol{F}^{\mathrm{p}}, \boldsymbol{Z}\right)+R C_{\mathrm{v}} T\left(1-\log \frac{T}{T_{0}}\right),
$$

where $W^{\mathrm{e}}$ is the elastic strain-energy density, $W^{\mathrm{p}}$ is the stored energy of cold work, $\boldsymbol{Z}$ is a collection of hardening variables depending on the material type (Lubliner, 1972), $C_{\mathrm{v}}$ is the specific heat per unit mass at constant volume and $T_{0}$ is a reference temperature. In this setting, the complete set of internal variables is $\left\{\boldsymbol{F}^{\mathrm{p}}, \boldsymbol{Z}\right\}$. The plastic deformation $\boldsymbol{F}^{\mathrm{p}}$ must define an invertible orientation-preserving local deformation, and hence the natural domain of $\boldsymbol{F}^{\mathrm{p}}$ is the multiplicative Lie group $G L_{+}(3, \mathbb{R})$. By material-frame indifference, it follows that $W^{\mathrm{e}}$ can only depend on $\boldsymbol{F}^{\mathrm{e}}$ through the elastic right-Cauchy Green deformation tensor:

$$
\boldsymbol{C}^{\mathrm{e}}=\boldsymbol{F}^{\mathrm{e} T} \boldsymbol{F}^{\mathrm{e}}=\boldsymbol{F}^{\mathrm{p}-T} \boldsymbol{C F}^{\mathrm{p}-1},
$$

whereupon (2.17) simplifies to

$$
A\left(\boldsymbol{F}, T, \boldsymbol{F}^{\mathrm{p}}, \boldsymbol{Z}\right)=W^{\mathrm{e}}\left(\boldsymbol{C}^{\mathrm{e}}, T\right)+W^{\mathrm{p}}\left(T, \boldsymbol{F}^{\mathrm{p}}, \boldsymbol{Z}\right)+R C_{\mathrm{v}} T\left(1-\log \frac{T}{T_{0}}\right) .
$$


Example 2.2 (Flow rules). An example of internal variable set arises in single-crystal plasticity, where $\boldsymbol{Z}=\left\{\gamma_{1}, \ldots, \gamma_{N}\right\}, \gamma_{\alpha}$ is the slip strain on slip system $\alpha$, and the rules of crystallographic slip require that (Ortiz and Stainier, 1999; Rice, 1971)

$$
\dot{\boldsymbol{F}}^{\mathrm{p}} \boldsymbol{F}^{\mathrm{p}-1}-\sum_{\alpha=1}^{N} \dot{\gamma}_{\alpha} \boldsymbol{s}_{\alpha} \otimes \boldsymbol{m}_{\alpha}=\mathbf{0},
$$

where $\left(\boldsymbol{s}_{\alpha}, \boldsymbol{m}_{\alpha}\right)$ are orthogonal unit vectors characteristic of the crystal class. Evidently, the flow rule (2.20) is of the general form (2.7). Another familiar example is provided by isotropic $J_{2}$-flow theory of plasticity (Ortiz and Stainier, 1999). In this case, $\boldsymbol{Z}=\left\{\varepsilon^{\mathrm{p}}\right\}$, where $\varepsilon^{\mathrm{p}}$ is the effective plastic strain, and the Prandtl-Reuss flow rule of plastic deformation requires

$$
\begin{aligned}
& \operatorname{tr}\left(\dot{\boldsymbol{F}}^{\mathrm{p}} \boldsymbol{F}^{\mathrm{p}-1}\right)=0, \\
& \left\|\dot{\boldsymbol{F}}^{\mathrm{p}} \boldsymbol{F}^{\mathrm{p}-1}\right\|^{2}-\frac{3}{2}\left|\dot{\varepsilon}^{\mathrm{p}}\right|^{2}=0,
\end{aligned}
$$

which are also of the general form (2.7).

In order to obtain a closed set of governing equations defining well-posed initial boundary-value problems the equilibrium relations summarized above need to be supplemented with appropriate kinetic relations enabling the determination of $\boldsymbol{P}^{\mathrm{v}}, \dot{\boldsymbol{Z}}$ and $\boldsymbol{H}$. We begin by considering a few special cases by way of example.

Example 2.3 (Rate-sensitivity). Consider a thermoelastic-plastic material such as described in example 2.1. Suppose that a kinetic potential $\psi(\boldsymbol{Y})$ exists such that

$$
\dot{\boldsymbol{Z}}=\partial_{\boldsymbol{Y}} \psi(\boldsymbol{Y}) .
$$

Introduce the dual potential

$$
\psi^{*}(\dot{\boldsymbol{Z}})=\sup _{\boldsymbol{Y}}\{\boldsymbol{Y} \cdot \dot{\boldsymbol{Z}}-\psi(\boldsymbol{Y})\}
$$

with the property that

$$
\boldsymbol{Y}=\partial_{\dot{\boldsymbol{Z}}} \psi^{*}(\dot{\boldsymbol{Z}}) \text {. }
$$

Example 2.4 (Newtonian viscosity). A continuum is said to possess Newtonian viscosity if the viscous part of the Cauchy stress tensor obeys the relation

$$
\boldsymbol{\sigma}^{\mathrm{v}}=\zeta \operatorname{tr}(\boldsymbol{d}) \boldsymbol{I}+2 \eta \boldsymbol{d},
$$

where

$$
\boldsymbol{d}=\operatorname{sym}\left(\dot{\boldsymbol{F}} \boldsymbol{F}^{-1}\right)
$$

is the rate of deformation tensor, and $\zeta$ and $\eta$ are viscosity parameters. The corresponding viscous part of the first Piola-Kirchhoff stress tensor is

$$
\boldsymbol{P}^{\mathrm{v}}=J \boldsymbol{\sigma}^{\mathrm{v}} \boldsymbol{F}^{-\mathrm{T}},
$$

where $J=\operatorname{det}(\boldsymbol{F})$ is the Jacobian determinant of deformation gradient $\boldsymbol{F}$. A simple calculation shows that the Newtonian viscosity law possesses the potential structure

$$
\boldsymbol{P}^{\mathrm{v}}=\partial_{\dot{\boldsymbol{F}}} \phi^{*}\left(\dot{\boldsymbol{F}} \boldsymbol{F}^{-1}\right),
$$


where the viscous potential per unit undeformed volume is

$$
\phi^{*}=J\left[\frac{\zeta}{2} \operatorname{tr}(\boldsymbol{d})^{2}+\eta \boldsymbol{d} \cdot \boldsymbol{d}\right] .
$$

Example 2.5 (Heat conduction). Assume that a Fourier potential $\chi(\boldsymbol{G})$ (also known as Biot's dissipation function (Biot, 1958)) exists such that

$$
\boldsymbol{H}=\partial_{\boldsymbol{G}} \chi(\boldsymbol{G}),
$$

where

$$
\boldsymbol{G}=-\frac{1}{T} \operatorname{Grad} T .
$$

A general kinetic potential is a function $\Delta(\dot{\boldsymbol{F}}, \dot{\boldsymbol{Z}}, \boldsymbol{G} ; \boldsymbol{F}, N, \boldsymbol{Z})$ such that

$$
\begin{gathered}
\boldsymbol{P}^{\mathrm{v}}=\partial_{\dot{\boldsymbol{F}}} \Delta(\dot{\boldsymbol{F}}, \dot{\boldsymbol{Z}}, \boldsymbol{G} ; \boldsymbol{F}, N, \boldsymbol{Z}), \\
\boldsymbol{Y}=\partial_{\dot{\boldsymbol{Z}}} \Delta(\dot{\boldsymbol{F}}, \dot{\boldsymbol{Z}}, \boldsymbol{G} ; \boldsymbol{F}, N, \boldsymbol{Z}), \\
-\boldsymbol{H}=\partial_{\boldsymbol{G}} \Delta(\dot{\boldsymbol{F}}, \dot{\boldsymbol{Z}}, \boldsymbol{G} ; \boldsymbol{F}, N, \boldsymbol{Z}),
\end{gathered}
$$

i.e., a function that acts as joint potential for the viscosity law, rate-sensitivity and the heat conduction law. For instance, for a material having uncoupled rate sensitivity, viscosity and heat conduction,

$$
\Delta=\psi^{*}+\phi^{*}-\chi,
$$

where $\psi^{*}, \phi^{*}$ and $\chi$ are the kinetic potential, viscous potential and Fourier potential, respectively, defined in the foregoing. Note that it is possible to define a dual general kinetic potential by way of a Legendre transform

$$
\Delta^{*}(\dot{\boldsymbol{F}}, \dot{\boldsymbol{Z}}, \boldsymbol{H} ; \boldsymbol{F}, N, \boldsymbol{Z})=\sup _{\boldsymbol{G}}[\Delta(\dot{\boldsymbol{F}}, \dot{\boldsymbol{Z}}, \boldsymbol{G} ; \boldsymbol{F}, N, \boldsymbol{Z})+\boldsymbol{H} \cdot \boldsymbol{G}]
$$

such that

$$
\boldsymbol{G}=\partial_{\boldsymbol{H}} \Delta^{*}(\dot{\boldsymbol{F}}, \dot{\boldsymbol{Z}}, \boldsymbol{H} ; \boldsymbol{F}, N, \boldsymbol{Z}) .
$$

Convexity of $\Delta^{*}$ then ensures verification of inequality (2.12) (positive dissipation).

Specific examples of the thermodynamic and kinetic potentials introduced in the foregoing are presented in Section 5.2.

\section{Variational principles for the rate problem}

We now seek to find a variational characterization of the rate problem, i.e., the problem of finding the rate of change of the state of a body given its current state and appropriate forcing and boundary conditions. Specifically, we wish to identify a functional whose critical or stationary points are the solutions of the rate problem.

\subsection{Thermoelastic materials}

To set the stage for the treatment of the general dissipative solid, we begin by considering the simpler case of a conducting thermoelastic solid. In this case, the internal 
energy density is of the form

$$
E=E(\boldsymbol{F}, N) \text {. }
$$

We shall find it convenient to carefully differentiate between the equilibrium temperature corresponding to the state $(\boldsymbol{F}, N)$, given by the equilibrium relation

$$
\Theta=\partial_{N} E(\boldsymbol{F}, N)
$$

and an external temperature field $T$. At equilibrium these two temperatures are equal everywhere, but this condition is not imposed a priori. It should be carefully noted that the notion of equilibrium invoked in differentiating between $\Theta$ and $T$ pertains to local thermodynamic equilibrium at material points and does not preclude the consideration of transient phenomena at the level of the entire body.

We wish to characterize variationally the solutions of the thermoelastic rate problem:

$$
\begin{aligned}
& T \dot{N}=-\operatorname{Div} \boldsymbol{H}+R Q \quad \text { in } B, \\
& \boldsymbol{H} \cdot \boldsymbol{N}=\bar{H} \quad \text { on } \partial_{N} B, \\
& T=\bar{T} \quad \text { on } \partial B \backslash \partial_{N} B, \\
& T=\Theta \quad \text { in } B .
\end{aligned}
$$

Here, $\bar{H}$ is the prescribed outward heat flux over the Neumann boundary $\partial_{N} B$; and $\bar{T}$ is the prescribed temperature over the Dirichlet boundary $\partial B \backslash \partial_{N} B$. The current state $(\boldsymbol{F}, N)$ of the solid is assumed given and the objective is to determine the unknown fields $(T, \dot{N})$. The weak form of $(3.3 \mathrm{a})-(3.3 \mathrm{~d})$ is

$$
\begin{aligned}
b[T, \dot{N}](\eta, n) \equiv & \int_{B} \boldsymbol{H} \cdot \operatorname{Grad} \frac{\eta}{T} \mathrm{~d} V+\int_{B} R Q \frac{\eta}{T} \mathrm{~d} V-\int_{\partial_{N} B} \bar{H} \frac{\eta}{T} \mathrm{~d} S \\
& +\int_{B}[(\Theta-T) n-\dot{N} \eta] \mathrm{d} V,
\end{aligned}
$$

$\eta$ and $n$ are admissible variations of $T$ and $\dot{N}$, respectively, i.e., variations such that

$$
\eta=0 \quad \text { on } \partial B \backslash \partial_{T} B .
$$

Suppose in addition that there exists a Fourier potential $\chi(\boldsymbol{G} ; \boldsymbol{F}, N)$ such that Fourier's law of heat conduction is of the form (2.30). Then, the weak form (3.4) becomes

$$
\begin{aligned}
b[T, \dot{N}](\eta, n) \equiv & \int_{B} \partial_{G} \chi \cdot \operatorname{Grad} \frac{\eta}{T} \mathrm{~d} V+\int_{B} R Q \frac{\eta}{T} \mathrm{~d} V-\int_{\partial_{N} B} \bar{H} \frac{\eta}{T} \mathrm{~d} S \\
& +\int_{B}[(\Theta-T) n-\dot{N} \eta] \mathrm{d} V,
\end{aligned}
$$

and, assuming sufficient differentiability, the second variation evaluates to

$$
\begin{aligned}
a[T, \dot{N}](\eta, n ; \xi, m) \equiv & D(b[T, \dot{N}](\eta, n))(\xi, m) \\
= & -\int_{B}\left\{\partial_{G G} \chi \cdot \operatorname{Grad} \frac{\eta}{T} \otimes \operatorname{Grad} \frac{\xi}{T}+\partial_{G} \chi \cdot \operatorname{Grad} \frac{\xi \eta}{T^{2}}\right\} \mathrm{d} V \\
& -\int_{B} R Q \frac{\xi \eta}{T^{2}} \mathrm{~d} V+\int_{\partial_{N} B} \bar{H} \frac{\xi \eta}{T^{2}} \mathrm{~d} S-\int_{B}(\xi n+\eta m) \mathrm{d} V,
\end{aligned}
$$


which, evidently, is symmetric in $(\xi, m)$ and $(\eta, n)$. From this symmetry it follows that the weak form $b$ is the first variation of the potential (cf., e.g., Marsden and Hughes, 1983)

$$
\Phi[T, \dot{N}]=\Phi_{0}+\int_{0}^{1} b[T(\lambda), \dot{N}(\lambda)]\left(\frac{\mathrm{d} T}{\mathrm{~d} \lambda}, \frac{\mathrm{d} \dot{N}}{\mathrm{~d} \lambda}\right) \mathrm{d} \lambda,
$$

where $(T(\lambda), \dot{N}(\lambda))$ is a path joining a referential state $\left(T_{0}, \dot{N}_{0}\right)$ to $(T, \dot{N})$ and $\Phi_{0}=$ $\Phi\left[T_{0}, \dot{N}_{0}\right]$ is referential datum. Therefore, it follows that the weak statement

$$
b[T, \dot{N}](\eta, n)=0,
$$

of the rate problem is equivalent to the stationarity principle

$$
\delta \Phi=0,
$$

i.e., $(T, \dot{N})$ is a solution of the rate problem if and only if it is a critical point of $\Phi$. The evaluation of formula (3.8) is straightforward and yields, explicitly,

$$
\begin{aligned}
\Phi[T, \dot{N}]= & \int_{B}\left[(\Theta-T) \dot{N}-\chi\left(-\frac{1}{T} \operatorname{Grad} T ; \boldsymbol{F}, N\right)\right] \mathrm{d} V \\
& +\int_{B} R Q \log \frac{T}{T_{0}} \mathrm{~d} V-\int_{\partial_{N} B} \bar{H} \log \frac{T}{T_{0}} \mathrm{~d} S,
\end{aligned}
$$

where we have set $\dot{N}_{0}=0$ and $\Phi_{0}=0$ without loss of generality. The stationarity principle (3.10) may be readily verified as follows. Taking variations with respect to admissible fields yields

$$
\begin{aligned}
& \int_{B}\left(-\dot{N} \eta+\boldsymbol{H} \cdot \operatorname{Grad} \frac{\eta}{T}\right) \mathrm{d} V+\int_{B} R Q \frac{\eta}{T} \mathrm{~d} V-\int_{\partial_{N} B} \bar{H} \frac{\eta}{T} \mathrm{~d} S=0, \\
& \int_{B}(\Theta-T) n \mathrm{~d} V=0 .
\end{aligned}
$$

Eq. (3.12b) requires that $\Theta=T$ at equilibrium. Integrating by parts Eq. (3.12a) yields

$$
\int_{B}\left(-\dot{N}-\frac{\operatorname{Div} \boldsymbol{H}}{T}+\frac{R Q}{T}\right) \eta \mathrm{d} V+\int_{\partial_{N} B}(\boldsymbol{H} \cdot \boldsymbol{N}-\bar{H}) \frac{\eta}{T} \mathrm{~d} S=0,
$$

which gives the heat equation in entropy form and the Neumann boundary conditions, as required.

Suppose now, by way of guiding example, that $\partial_{N} B=\emptyset, Q$ is square-integrable over $B$, $\chi(\boldsymbol{G} ; \boldsymbol{F}, N)$ is quadratic and strictly convex in $\boldsymbol{G}$. Then, for every square-integrable $\dot{N}$ the functional $\Phi[T, \dot{N}]$ attains a unique maximum and has no other extrema (cf., e.g., Dal Maso, 1993). In addition, the reduced functional

$$
\Phi[\dot{N}]=\sup _{T} \Phi[T, \dot{N}]
$$

is convex. The remaining problem of determining the field $\dot{N}$ may thus be expressed as the minimization problem

$$
\inf _{\dot{N}} \Phi[\dot{N}] .
$$


Alternatively, both variational problems may be collected jointly as

$$
\inf _{\dot{N}} \sup _{T} \Phi[T, \dot{N}] .
$$

It should be noted that, owing to the dependence of $\Phi[T, \dot{N}]$ on gradients of $T$, the reduced functional $\Phi[\dot{N}]$ is strongly nonlocal. For the example under consideration, (3.15) or (3.16) may alternatively be used instead of the stationarity condition (3.10). For general materials we may still postulate the saddle-point (3.16) as the variational statement of the rate problem, in the expectation that, among all critical points of $\Phi[T, \dot{N}]$, its saddle points define the stable solutions of the problem.

The properties of the solutions of (3.15) and (3.16) follow from standard theory (cf., e.g., Dal Maso, 1993) and may be summarized as follows. For definiteness, assume that $B$ is open and bounded, $\partial_{N} B=\emptyset$, corresponding to Dirichlet boundary conditions, and rephrase the problem in terms of the field $u=\log \left(T / T_{0}\right)$, which takes values over the entire real line $\mathbb{R}$. If $\chi$ is convex in $\nabla u$ and grows as a power $|\nabla u|^{p}, 1<p<+\infty$, then $\Phi[\cdot, \dot{N}]$ attains its supremum $\Phi[\dot{N}]$ in the Sobolev space $W^{1, p}(B)$. If, in addition, $\chi$ is strictly convex in $\nabla u$ then the temperature field is determined uniquely. If $\chi$ is continuous but not convex in $\nabla u$ and grows as a power $|\nabla u|^{p}$, then the supremum of $\Phi[\cdot, \dot{N}]$ is attained by its relaxation. The relaxation of $\Phi[\cdot, \dot{N}]$ is obtained simply by replacing $\chi(\nabla u ; \boldsymbol{F}, N)$ by its convex envelope $\chi^{* *}(\nabla u ; \boldsymbol{F}, N)$, the largest convex function majorized by $\chi(\nabla u ; \boldsymbol{F}, N)$. The reduced potential $\Phi[\dot{N}]$ is the supremum of a collection of linear functionals and, therefore, is convex, lower semi-continuous and coercive in $W^{1, q}(B)$, with $1 / p+1 / q=1$. Hence, $\Phi[\dot{N}]$ has a minimum point in $W^{1, q}(B)$.

An alternative avenue for arriving at the preceding variational problems is as follows. Begin by introducing the functional of temperature

$$
\Psi[\Theta]=-\int_{B} \chi(\boldsymbol{G} ; \boldsymbol{F}, N) \mathrm{d} V+\int_{B} R Q \log \frac{\Theta}{T_{0}} \mathrm{~d} V-\int_{\partial_{N} B} \bar{H} \log \frac{\Theta}{T_{0}} \mathrm{~d} S,
$$

where we set $\boldsymbol{G}=-\Theta^{-1}$ Grad $\Theta$. Define its subdifferential, or functional derivative, $\partial \Psi[\Theta]$ with respect to $\Theta$ through the identity

$$
\langle\partial \Psi[\Theta], \eta\rangle=D \Psi[\Theta](\eta),
$$

where $D \Psi[\Theta](\eta)$ is the Frechet derivative of $\Psi$ (Rudin, 1973) and we assume differentiability as required. A straightforward calculation gives, explicitly,

$$
\partial \Psi[\Theta]=\frac{R Q}{\Theta}-\frac{\operatorname{Div} \boldsymbol{H}}{\Theta} .
$$

The energy equation can now be expressed in the equation-of-evolution form (cf., e.g., Barbu, 1976)

$$
\dot{N}=\partial \Psi[\Theta] .
$$

Let

$$
\Psi^{*}[\dot{N}]=\sup _{T}\left\{\Psi[T]-\int_{B} T \dot{N} \mathrm{~d} V\right\}
$$


be the dual functional (Ekelan and Temam, 1976). Then, (3.20) is equivalent to the minimum problem

$$
\inf _{\dot{N}}\left\{\Psi^{*}[\dot{N}]+\int_{B} \Theta \dot{N} \mathrm{~d} V\right\} .
$$

Combining (3.21) and (3.22) we arrive at the variational problem

$$
\inf _{\dot{N}} \sup _{T}\left\{\int_{B}(\Theta-T) \dot{N} \mathrm{~d} V+\Psi[T]\right\} .
$$

But

$$
\Phi[T, \dot{N}]=\int_{B}(\Theta-T) \dot{N} \mathrm{~d} V+\Psi[T]
$$

and, consequently, (3.23) is equivalent to (3.16).

Conceptually, the most succinct and direct variational characterization of the rate problem is furnished by the minimum principle (3.15), which characterizes the entropy rate field $\dot{N}$ as the minimizer of the single-field functional $\Phi[\dot{N}]$. In practice this characterization has the drawback that, as noted earlier, the functional $\Phi[\dot{N}]$ is strongly nonlocal and involves long-range interactions. By contrast, the advantage of the two-field variational principle (3.16) is that the functional $\Phi[T, \dot{N}]$ is local.

\subsection{Extension to general materials}

We now proceed to extend the preceding variational framework to general materials. To this end, we consider materials characterized by internal energy density (2.8a), equilibrium relations (2.9a), (2.11) and (2.9b); and potential kinetic relations (2.32a)-(2.32c). As in the preceding example, we shall find it necessary to carefully differentiate between the equilibrium temperature corresponding to the state $(\boldsymbol{F}, N, \boldsymbol{Z})$, given by the equilibrium relation

$$
\Theta=\partial_{N} E(\boldsymbol{F}, N, \boldsymbol{Z}),
$$

and an external temperature field $T$. At equilibrium these two temperatures are equal everywhere, but this condition is not imposed a priori. We wish to characterize variationally the solutions of the general rate problem:

$$
\begin{aligned}
& \operatorname{Div}\left(\boldsymbol{P}^{\mathrm{e}}+\partial_{\dot{\boldsymbol{F}}} \Delta\right)+R \boldsymbol{B}=\mathbf{0} \quad \text { in } B, \\
& \left(\boldsymbol{P}^{\mathrm{e}}+\partial_{\dot{\boldsymbol{F}}} \Delta\right) \cdot \boldsymbol{N}=\overline{\boldsymbol{T}} \quad \text { on } \partial_{T} B, \\
& \dot{\boldsymbol{\varphi}}=\dot{\overline{\boldsymbol{\varphi}}} \quad \text { on } \partial B \backslash \partial_{T} B, \\
& T \dot{N}=\boldsymbol{P}^{\mathrm{v}} \cdot \dot{\boldsymbol{F}}+\boldsymbol{Y} \cdot \dot{\boldsymbol{Z}}-\operatorname{Div} \boldsymbol{H}+R Q \quad \text { in } B, \\
& -\partial_{\boldsymbol{G}} \Delta \cdot \boldsymbol{N}=\bar{H} \quad \text { on } \partial_{N} B, \\
& T=\bar{T} \quad \text { on } \partial B \backslash \partial_{T} N,
\end{aligned}
$$




$$
\begin{aligned}
& T=\Theta \quad \text { in } B, \\
& \boldsymbol{Y}=\partial_{\dot{\boldsymbol{Z}}} \Delta \quad \text { in } B .
\end{aligned}
$$

Here, $\partial_{T} B$ and $\partial_{N} B$ denote the traction and Neumann boundaries where the tractions and heat flux are prescribed, respectively; $\overline{\boldsymbol{T}}$ are the applied tractions over the traction boundary; $\dot{\bar{\varphi}}$ are the prescribed velocities over the displacement boundary; $\bar{H}$ is the prescribed outward heat flux over the Neumann boundary; $\bar{T}$ is the prescribed temperature over the Dirichlet boundary; and we set $\boldsymbol{G}=-T^{-1} \mathrm{Grad} T$ in the computation of the kinetic potential $\Delta$. In the general rate problem just stated the current local state $(\boldsymbol{F}, N, \boldsymbol{Z})$ is given and the objective is to determine the unknown rates $(\dot{\boldsymbol{F}}, \dot{N}, \dot{\boldsymbol{Z}})$. It should be carefully noted that $\boldsymbol{P}^{\mathrm{e}}, \Theta$ and $\boldsymbol{Y}$ are functions of state and, since the local state is fixed, they appear in the general rate problem as known fields. In order to simplify the notation, throughout this section we shall omit all dependencies of the response functions on the fixed state $(\boldsymbol{F}, N, \boldsymbol{Z})$.

The general rate (3.26a)-(3.26h) does not have an obvious variational structure. A naive weak statement of Eqs. (3.26a)-(3.26h), e.g., obtained by multiplying each of the field equations by admissible variations of the fields, leads to a weak form that does not derive from a potential. Thus, the ability to formulate the general rate problem in variational form hinges on the identification of an integration factor that delivers the requisite symmetry of the Dirichlet form. We shall specifically consider integrating factors obtained by a time rescaling of the kinetic equations. We accomplish this rescaling by computing the kinetic potential as

$$
\Delta=\Delta(f(T, \Theta) \dot{\boldsymbol{F}}, f(T, \Theta) \dot{Z}, \boldsymbol{G})
$$

which corresponds to resetting the time scale according to the mapping $\mathrm{d} t \rightarrow \mathrm{d} t / f(T, \Theta)$ in all rate processes. The function $f(T, \Theta)$ is required to satisfy the identity

$$
f(T, T)=1 .
$$

This normalization condition ensures that the physical time scale is recovered at equilibrium. The rescaled weak form is

$$
\begin{aligned}
b[\dot{\boldsymbol{\varphi}}, & T, \dot{N}, \dot{\boldsymbol{Z}}](\boldsymbol{v}, \eta, n, \boldsymbol{\beta}) \\
= & \int_{B}\left[\left(\boldsymbol{P}^{\mathrm{e}}+f \partial_{\dot{\boldsymbol{F}}} \Delta\right) \cdot \operatorname{Grad} \boldsymbol{v} \mathrm{d} V-\int_{B} R \boldsymbol{B} \cdot \boldsymbol{v} \mathrm{d} V-\int_{\partial_{T} B} \overline{\boldsymbol{T}} \cdot \boldsymbol{v} \mathrm{d} S\right. \\
& +\int_{B}\left[-\eta \dot{N}+\left(f \partial_{\dot{\boldsymbol{F}}} \Delta \cdot \dot{\boldsymbol{F}}+f \partial_{\dot{\boldsymbol{Z}}} \Delta \cdot \dot{\boldsymbol{Z}}\right) \frac{\eta}{T}-\partial_{\boldsymbol{G}} \Delta \cdot \operatorname{Grad} \frac{\eta}{T}\right] \mathrm{d} V \\
& +\int_{B} R Q \frac{\eta}{T} \mathrm{~d} V-\int_{\partial_{N} B} \bar{H} \frac{\eta}{T} \mathrm{~d} S \\
& +\int_{B}(\Theta-T) n \mathrm{~d} V-\int_{B}\left(\boldsymbol{Y}-f \partial_{\dot{\boldsymbol{Z}}} \Delta\right) \cdot \boldsymbol{\beta} \mathrm{d} V,
\end{aligned}
$$

which can be expressed as the sum

$$
b=b_{I}+b_{I I},
$$


where

$$
\begin{aligned}
b_{I}[\dot{\boldsymbol{\varphi}}, & T, \dot{N}, \dot{\boldsymbol{Z}}](\boldsymbol{v}, \eta, n, \boldsymbol{\beta}) \\
= & \int_{B}\left[\left(\boldsymbol{P}^{\mathrm{e}}+f \partial_{\dot{\boldsymbol{F}}} \Delta\right) \cdot \operatorname{Grad} \boldsymbol{v} \mathrm{d} V-\int_{B} R \boldsymbol{B} \cdot \boldsymbol{v} \mathrm{d} V-\int_{\partial_{T} B} \overline{\boldsymbol{T}} \cdot \boldsymbol{v} \mathrm{d} S\right. \\
& +\int_{B}\left[-\eta \dot{N}+\left(\partial_{\dot{\boldsymbol{F}}} \Delta \cdot \dot{\boldsymbol{F}}+\partial_{\dot{\boldsymbol{Z}}} \Delta \cdot \dot{\boldsymbol{Z}}\right) \partial_{T} f \eta-\partial_{\boldsymbol{G}} \Delta \cdot \mathrm{Grad} \frac{\eta}{T}\right] \mathrm{d} V \\
& +\int_{B} R Q \frac{\eta}{T} \mathrm{~d} V-\int_{\partial_{N} B} \bar{H} \frac{\eta}{T} \mathrm{~d} S \\
& +\int_{B}(\Theta-T) n \mathrm{~d} V-\int_{B}\left(\boldsymbol{Y}-f \partial_{\dot{Z}^{\Delta}}\right) \cdot \boldsymbol{\beta} \mathrm{d} V,
\end{aligned}
$$

and

$$
b_{I I}[\dot{\boldsymbol{\varphi}}, T, \dot{N}, \dot{\boldsymbol{Z}}](\boldsymbol{v}, \eta, n, \boldsymbol{\beta})=\int_{B}\left(\partial_{\dot{\boldsymbol{F}}} \Delta \cdot \dot{\boldsymbol{F}}+\partial_{\dot{\boldsymbol{Z}}} \Delta \cdot \dot{\boldsymbol{Z}}\right)\left(\frac{f}{T}-\partial_{T} f\right) \eta
$$

A lengthy but straightforward calculation shows that the linearization of $b_{I}$ is symmetric. Hence, the Dirichlet form corresponding to $b$ is itself symmetric if and only if

$$
\partial_{T} f=\frac{f}{T} \text {. }
$$

The general solution of this equation is

$$
f=C(\Theta) T \text {. }
$$

In addition, the normalization condition (3.28) requires that $C(\Theta)=\Theta^{-1}$, whereupon the integrating factor is identified uniquely as

$$
f=\frac{T}{\Theta} \text {. }
$$

The corresponding potential now follows from the formula

$$
\Phi[\dot{\boldsymbol{\varphi}}, T, \dot{N}, \dot{Z}]=\Phi_{0}+\int_{0}^{1} b[\dot{\boldsymbol{\varphi}}(\lambda), T(\lambda), \dot{N}(\lambda), \dot{Z}(\lambda)]\left(\frac{\mathrm{d} \dot{\boldsymbol{\varphi}}}{\mathrm{d} \lambda}, \frac{\mathrm{d} T}{\mathrm{~d} \lambda}, \frac{\mathrm{d} \dot{N}}{\mathrm{~d} \lambda}, \frac{\mathrm{d} \dot{Z}}{\mathrm{~d} \lambda}\right) \mathrm{d} \lambda,
$$

where $(\dot{\varphi}(\lambda), T(\lambda), \dot{N}(\lambda), \dot{Z}(\lambda))$ is a path joining a referential state $\left(\dot{\varphi}_{0}, T_{0}, \dot{N}_{0}, \dot{Z}_{0}\right)$ to $(\dot{\boldsymbol{\varphi}}, T, \dot{N}, \dot{\boldsymbol{Z}})$ and $\Phi_{0}=\Phi\left[\dot{\boldsymbol{\varphi}}_{0}, T_{0}, \dot{N}_{0}, \dot{Z}_{0}\right]$ is the referential datum. Eq. (3.36) can be evaluated simply, with the result

$$
\begin{aligned}
\Phi[\dot{\boldsymbol{\varphi}}, T, \dot{N}, \dot{Z}]= & \int_{B}\left[\dot{E}-T \dot{N}+\Delta\left(\frac{T}{\Theta} \dot{F}, \frac{T}{\Theta} \dot{\boldsymbol{Z}},-\frac{1}{T} \operatorname{Grad} T\right)\right] \mathrm{d} V \\
& -\int_{B} R \boldsymbol{B} \cdot \dot{\boldsymbol{\varphi}} \mathrm{d} V-\int_{\partial_{T} B} \overline{\boldsymbol{T}} \cdot \dot{\boldsymbol{\varphi}} \mathrm{d} S \\
& +\int_{B} R Q \log \frac{T}{T_{0}} \mathrm{~d} V-\int_{\partial_{N} B} \bar{H} \log \frac{T}{T_{0}} \mathrm{~d} S .
\end{aligned}
$$

It, therefore, follows that the weak statement

$$
b[\dot{\boldsymbol{\varphi}}, T, \dot{N}, \dot{\boldsymbol{Z}}](\boldsymbol{v}, \eta, n, \boldsymbol{\beta})=0
$$

of the general rate problem is equivalent to the stationarity principle

$$
\delta \Phi=0,
$$


i.e., assuming sufficient differentiability, $(\dot{\boldsymbol{\varphi}}, T, \dot{N}, \dot{Z})$ is a solution of the general rate problem if and only if it is a critical point of $\Phi$. As in the case of the thermoelastic solid, the stationarity principle (3.39) may be readily verified by taking variations of the potential (3.37) directly. This leads to the weak statement $b_{I}=0$, with $b_{I}$ as in (3.31), which, evidently, is equivalent to (3.26a)-(3.26h). In particular, the stationarity principle requires $T=\Theta$ at equilibrium.

As in the thermoelastic rate problem, we may wish to restrict attention to the problem of determining the extrema of $\Phi$ in the expectation that they correspond to the stable states of the solid. Under this postulate of stability, the thermoelastic variational principle (3.16) generalizes to

$$
\inf _{\dot{\varphi}, \dot{Z}, \dot{N}} \sup _{T} \Phi[\dot{\varphi}, T, \dot{N}, \dot{Z}] .
$$

As in the thermoelastic rate problem, we may also define the reduced-albeit nonlocalpotential

$$
\Phi[\dot{\boldsymbol{\varphi}}, \dot{N}, \dot{Z}]=\sup _{T} \Phi[\dot{\boldsymbol{\varphi}}, T, \dot{N}, \dot{Z}]
$$

whereupon (3.40) reduces to the minimum problem

$$
\inf _{\dot{\varphi}, \dot{Z}, \dot{N}} \Phi[\dot{\varphi}, \dot{N}, \dot{Z}]
$$

As for the thermoelastic rate problem, conditions guaranteeing existence and uniqueness of the rate fields are supplied by standard theory (cf., e.g., Dal Maso, 1993).

\section{Variational principles for the incremental problem}

In this section, we proceed to establish a time-discretized version of the variational problems formulated in the foregoing. Time-discretization is of interest in analysis and in computation. Thus, in analysis, time-discretization is used to reduce time-dependent problems to a sequence of incremental problems each characterized by a minimum principle. For instance, time-discretization has been employed to formulate incremental minimum principles for plasticity that establish a connection between non-attainment and the formation of microstructures (Ortiz and Repetto, 1999; Ortiz et al., 2000; Carstensen et al., 2002; Aubry and Ortiz, 2003). In addition, time-discretization is a key step in the numerical implementation of constitutive equations, e.g., within a finite-element framework. From this latter perspective, the aim of variational updates is to formulate timediscretizations of the constitutive relations that lead to an incremental problem having a variational structure. The variational updates for general dissipative solids presented in this section generalize the isothermal variational updates of Ortiz and Stainier (1999).

\subsection{Minimizing paths}

Formally, the time-discretized incremental variational problem can be derived by recourse to minimizing paths, in the vein of deformation theories of plasticity (cf. Martin and Ponter, 1966; Maier, 1969; Soechting and Lance, 1969; Carter and Martin, 1976, for the traditional view on deformation theories; cf. Ortiz and Martin, 1989; Ortiz and Repetto, 1999 for more recent accounts on minimizing paths). Here we envision a sequence 
of times $t_{0}, \ldots, t_{n}, t_{n+1}, \ldots$, and seek to characterize the state $(\varphi, T, N, \boldsymbol{Z})$ of the solid at those times. In particular, assume that the state $\left(\boldsymbol{\varphi}_{n}, T_{n}, N_{n}, \boldsymbol{Z}_{n}\right)$ is known. Then, we wish to consistently approximate the state $\left(\boldsymbol{\varphi}_{n+1}, T_{n+1}, N_{n+1}, Z_{n+1}\right)$ at time $t_{n+1}$ as the solution of an extremum problem. By a consistent approximation we mean that the limits of the divided differences $\left[\left(\varphi_{n+1}-\varphi_{n}\right) / \Delta t,\left(T_{n+1}-T_{n}\right) / \Delta t,\left(N_{n+1}-N_{n}\right) / \Delta t,\left(\boldsymbol{Z}_{n+1}-\boldsymbol{Z}_{n}\right) / \Delta t\right)$ as $\Delta t=t_{n+1}-t_{n}$ tends to zero satisfy the rate field equations.

To this end, introduce the incremental functional

$$
\Phi_{n}\left[\boldsymbol{\varphi}_{n+1}, T_{n+1}, N_{n+1}, \boldsymbol{Z}_{n+1}\right]=\inf _{\text {paths }} \int_{t_{n}}^{t_{n+1}} \Phi[\dot{\boldsymbol{\varphi}}, T, \dot{N}, \dot{\boldsymbol{Z}}] \mathrm{d} t,
$$

where the subscript $n$ signifies that $\Phi_{n}\left[\boldsymbol{\varphi}_{n+1}, T_{n+1}, N_{n+1}, Z_{n+1}\right]$ depends parametrically on the initial state $\left(\varphi_{n}, T_{n}, N_{n}, Z_{n}\right)$ at time $t_{n}$, and the minimum is taken over all admissible paths joining $\left(\boldsymbol{\varphi}_{n}, T_{n}, N_{n}, \boldsymbol{Z}_{n}\right)$ at time $t_{n}$ to $\left(\boldsymbol{\varphi}_{n+1}, T_{n+1}, N_{n+1}, \boldsymbol{Z}_{n+1}\right)$ at time $t_{n+1}$. The fundamental properties of the incremental potential $\Phi_{n}$ may be ascertained as follows. Begin by collecting all forcing terms within the functional

$$
G(\dot{\boldsymbol{\varphi}}, T) \equiv-\int_{B} R \boldsymbol{B} \cdot \dot{\boldsymbol{\varphi}} \mathrm{d} V-\int_{\partial_{T} B} \overline{\boldsymbol{T}} \cdot \dot{\boldsymbol{\varphi}} \mathrm{d} S+\int_{B} R Q \log \frac{T}{T_{0}} \mathrm{~d} V-\int_{\partial_{N} B} \bar{H} \log \frac{T}{T_{0}} \mathrm{~d} S
$$

for economy of notation. Then (4.1) can be written as

$$
\Phi_{n}\left[\boldsymbol{\varphi}_{n+1}, T_{n+1}, N_{n+1}, Z_{n+1}\right]=\inf _{\text {paths }} \int_{t_{n}}^{t_{n+1}}\left\{\int_{B}(\dot{E}-T \dot{N}+\Delta) \mathrm{d} V+G(\dot{\boldsymbol{\varphi}}, T)\right\} \mathrm{d} t,
$$

where $\Delta$ is evaluated as in (3.37). Integration of the perfect differential $\dot{E}$ gives

$$
\begin{aligned}
\Phi_{n} & {\left[\boldsymbol{\varphi}_{n+1}, T_{n+1}, N_{n+1}, Z_{n+1}\right] } \\
& =\int_{B}\left(E_{n+1}-E_{n}\right) \mathrm{d} V+\inf _{\text {paths }} \int_{t_{n}}^{t_{n+1}}\left\{\int_{B}(-T \dot{N}+\Delta) \mathrm{d} V+G(\dot{\varphi}, T)\right\} \mathrm{d} t .
\end{aligned}
$$

Taking variations with respect to $T$ and enforcing stationarity gives

$$
\begin{aligned}
& \dot{N}=\frac{1}{\Theta} \partial_{\dot{\boldsymbol{F}}} \Delta \cdot \dot{\boldsymbol{F}}+\frac{1}{\Theta} \partial_{\dot{\boldsymbol{Z}}} \Delta \cdot \dot{\boldsymbol{Z}}-\frac{1}{T} \operatorname{Div} \boldsymbol{H}+\frac{1}{T} R Q \quad \text { in } B, \\
& -\partial_{\boldsymbol{G}} \Delta \cdot \boldsymbol{N}=\bar{H} \quad \text { on } \partial_{N} B
\end{aligned}
$$

in the interval $t \in\left[t_{n}, t_{n+1}\right]$, which shows that the minimizing temperature path satisfies the heat equation in entropy form. Taking variations with respect to the remaining variables in turn and enforcing stationarity gives

$$
\begin{aligned}
& \int_{B} \boldsymbol{P}_{n+1}^{\mathrm{e}} \cdot \delta \boldsymbol{F}_{n+1} \mathrm{~d} V+\int_{t_{n}}^{t_{n+1}} \int_{B}\left[\frac{T}{\Theta} \partial_{\dot{\boldsymbol{F}}} \Delta \cdot \delta \dot{\boldsymbol{F}}+\partial_{\boldsymbol{F}} \Delta \cdot \delta \boldsymbol{F}\right] \mathrm{d} V \mathrm{~d} t \\
& +\int_{t_{n}}^{t_{n+1}}\left[\int_{B} R \boldsymbol{B} \cdot \delta \dot{\boldsymbol{\varphi}} \mathrm{d} V+\int_{\partial_{T} B} \overline{\boldsymbol{T}} \cdot \delta \dot{\boldsymbol{\varphi}} \mathrm{d} S\right] \mathrm{d} t=0, \\
& \int_{B} \Theta_{n+1} \cdot \delta N_{n+1} \mathrm{~d} V+\int_{t_{n}}^{t_{n+1}} \int_{B}\left(-T \delta \dot{N}+\partial_{N} \Delta \delta N\right) \mathrm{d} V \mathrm{~d} t=0,
\end{aligned}
$$




$$
-\int_{B} \boldsymbol{Y}_{n+1} \cdot \delta \boldsymbol{Z}_{n+1} \mathrm{~d} V+\int_{t_{n}}^{t_{n+1}} \int_{B}\left(\frac{T}{\Theta} \partial_{\dot{Z}} \Delta \cdot \delta \dot{Z}+\partial_{\boldsymbol{Z}} \Delta \cdot \delta \boldsymbol{Z}\right) \mathrm{d} V \mathrm{~d} t=0 .
$$

Integration by parts with respect to time and localization of the result finally gives

$$
\begin{aligned}
& \operatorname{Div}\left[-\frac{\mathrm{d}}{\mathrm{d} t}\left(\frac{T}{\Theta} \partial_{\dot{\boldsymbol{F}}} \Delta\right)+\partial_{\boldsymbol{F}} \Delta\right]+R \dot{\boldsymbol{B}}=0 \quad \text { in } B, \\
& {\left[-\frac{\mathrm{d}}{\mathrm{d} t}\left(\frac{T}{\Theta} \partial_{\dot{\boldsymbol{F}}} \Delta\right)+\partial_{\boldsymbol{F}} \Delta\right] \cdot \boldsymbol{N}=\dot{\overline{\boldsymbol{T}}} \text { in } \partial_{T} B,} \\
& \dot{T}+\partial_{N} \Delta=0 \quad \text { in } B, \\
& -\frac{\mathrm{d}}{\mathrm{d} t}\left(\frac{T}{\Theta} \partial_{\dot{Z}} \Delta\right)+\partial_{\boldsymbol{Z}} \Delta=0 \quad \text { in } B
\end{aligned}
$$

in the interval $t \in\left[t_{n}, t_{n+1}\right]$, and

$$
\begin{aligned}
& \operatorname{Div}\left(\boldsymbol{P}_{n+1}^{\mathrm{e}}+\frac{T_{n+1}}{\Theta_{n+1}} \partial_{\dot{\boldsymbol{F}}_{n+1}} \Delta_{n+1}\right)+R \boldsymbol{B}_{n+1}=0 \text { in } B, \\
& \left(\boldsymbol{P}_{n+1}^{\mathrm{e}}+\frac{T_{n+1}}{\Theta_{n+1}} \partial_{\dot{\boldsymbol{F}}_{n+1}} \Delta_{n+1}\right) \cdot \boldsymbol{N}=\overline{\boldsymbol{T}}_{n+1} \quad \text { on } \partial_{T} B, \\
& \Theta_{n+1}-T_{n+1}=0 \quad \text { in } B \\
& \boldsymbol{Y}_{n+1}=\frac{T_{n+1}}{\Theta_{n+1}} \partial_{\dot{\boldsymbol{Z}}_{n+1}} \Delta_{n+1} \quad \text { in } B
\end{aligned}
$$

at time $t_{n+1}$. Eqs. (4.7a)-(4.7d), in conjunction with (4.5a) and (4.5b), determine the minimizing paths. The remaining Eqs. (4.8a)-(4.8d) are then the Euler-Lagrange equations of the functional $\Phi_{n}$. Evidently, these Euler-Lagrange equations are the rate field equations of the general dissipative material at time $t_{n+1}$. Thus, we have shown that the critical points of $\Phi_{n}$ satisfy the rate field equations at time $t_{n+1}$ with rates computed from the corresponding minimizing paths.

An additional requirement of stability leads to the incremental extremum problem

$$
\inf _{\boldsymbol{\varphi}_{n+1}, N_{n+1}, \boldsymbol{Z}_{n+1}} \sup _{T_{n+1}} \Phi_{n}\left[\boldsymbol{\varphi}_{n+1}, T_{n+1}, N_{n+1}, \boldsymbol{Z}_{n+1}\right]
$$

whereby the stable states at time $t_{n+1}$ are identified with the extrema of $\Phi_{n}$. In particular, minimization with respect to the internal state $\boldsymbol{Z}_{n+1}$ yields a reduced potential $\Phi_{n}\left[\varphi_{n+1}, T_{n+1}, N_{n+1}\right]$, and the subsequent extremum problem is indistinguishable from that of a conducting thermoelastic material. However, it should be carefully noted that the incremental functional $\Phi_{n}$ reflects both the energetics as well as the kinetics of the material. A manifestation of the kinetic character of $\Phi_{n}$ is its parametric dependence on the initial conditions at time $t_{n}$. Alternatively, we may regard the incremental functional $\Phi_{n}$ as changing between time steps. This incremental nature of $\Phi_{n}$ allows for irreversible behavior, path dependency and hysteresis, as required. 


\subsection{A difference scheme}

Despite the conceptual appeal of minimizing paths, their explicit determination can only be effected in simple cases (Ortiz and Martin, 1989). In calculations, it suffices to identify any convenient incremental potential $\Phi_{n}$ which is consistent with the field equations. An example of a family of consistent incremental potentials is furnished by

$$
\begin{aligned}
\Phi_{n} & {\left[\boldsymbol{\varphi}_{n+1}, T_{n+1}, N_{n+1}, Z_{n+1}\right] } \\
= & \int_{B}\left[\left(E_{n+1}-E_{n}\right)-T_{n+1}\left(N_{n+1}-N_{n}\right)+\Delta t \Delta_{n+1}\right] \mathrm{d} V \\
& -\int_{B} R \boldsymbol{B}_{n+1} \cdot\left(\boldsymbol{\varphi}_{n+1}-\boldsymbol{\varphi}_{n}\right) \mathrm{d} V-\int_{\partial_{T} B} \overline{\boldsymbol{T}}_{n+1} \cdot\left(\boldsymbol{\varphi}_{n+1}-\boldsymbol{\varphi}_{n}\right) \mathrm{d} S \\
& +\int_{B} \Delta t R Q_{n+1} \log \frac{T_{n+1}}{T_{n}} \mathrm{~d} V-\int_{\partial_{T} B} \Delta t \bar{H}_{n+1} \log \frac{T_{n+1}}{T_{n}} \mathrm{~d} S,
\end{aligned}
$$

where we write

$$
\Delta_{n+1}=\Delta\left(\frac{T_{n+1}}{T_{n}} \dot{\boldsymbol{F}}_{n+1}, \frac{T_{n+1}}{T_{n}} \dot{\boldsymbol{Z}}_{n+1}, \boldsymbol{G}_{n+1} ; \boldsymbol{F}_{n+1}, N_{n+1}, \boldsymbol{Z}_{n+1}\right)
$$

and

$$
\begin{aligned}
\dot{\boldsymbol{F}}_{n+1} & =\frac{\boldsymbol{F}_{n+1}-\boldsymbol{F}_{n}}{\Delta t}, \\
\dot{\boldsymbol{Z}}_{n+1} & =\frac{\boldsymbol{Z}_{n+1}-\boldsymbol{Z}_{n}}{\Delta t}, \\
\boldsymbol{G}_{n+1} & =- \text { Grad } \log \frac{T_{n+1}}{T_{n}} .
\end{aligned}
$$

The incremental functional (4.10) may be regarded as a backward-Euler approximation of (4.3). Other approximations, e.g., based on the trapezoidal or midpoint rules of integration, may be formulated likewise, but these enhancements will not be pursued here.

The consistency of the scheme can be verified as follows. Taking variations and enforcing stationarity yields

$$
\begin{aligned}
& \operatorname{Div}\left(\boldsymbol{P}_{n+1}^{\mathrm{e}}+\frac{T_{n+1}}{T_{n}} \partial_{\dot{\boldsymbol{F}}_{n+1}} \Delta_{n+1}\right)+R \boldsymbol{B}_{n+1}=O(\Delta t) \text { in } B, \\
& \left(\boldsymbol{P}_{n+1}^{\mathrm{e}}+\frac{T_{n+1}}{T_{n}} \partial_{\dot{\boldsymbol{F}}_{n+1}} \Delta_{n+1}\right) \cdot \boldsymbol{N}-\overline{\boldsymbol{T}}_{n+1}=O(\Delta t) \quad \text { on } \partial_{T} B, \\
& \frac{N_{n+1}-N_{n}}{\Delta t}=\frac{1}{T_{n}} \partial_{\dot{\boldsymbol{F}}_{n+1}} \Delta \cdot \dot{\boldsymbol{F}}_{n+1}+\frac{1}{T_{n}} \partial_{\dot{\boldsymbol{Z}}_{n+1}} \Delta \cdot \dot{\boldsymbol{Z}}_{n+1} \\
& \quad-\frac{1}{T_{n+1}} \operatorname{Div} \partial_{\boldsymbol{G}_{n+1}} \Delta+\frac{1}{T_{n+1}} R Q_{n+1} \quad \text { in } B, \\
& -\partial_{\boldsymbol{G}_{n+1}} \Delta_{n+1} \cdot \boldsymbol{N}=\bar{H}_{n+1} \quad \text { on } \partial_{N} B, \\
& \Theta_{n+1}-T_{n+1}=O(\Delta t) \quad \text { in } B,
\end{aligned}
$$




$$
-\boldsymbol{Y}_{n+1}+\frac{T_{n+1}}{T_{n}} \partial_{\dot{Z}_{n+1}} \Delta_{n+1}=O(\Delta t) \text { in } B
$$

Evidently, all rate field equations at $t_{n}$ are recovered as $\Delta t \rightarrow 0$, which proves the consistency of the scheme. The incremental problem defined by potential (4.10) is now formally identical to (4.9). The corresponding Euler-Lagrange equations may now be regarded as time discretizations of the rate field equations.

\subsection{Variational updates}

It bears emphasis that the dependence of the rate functional $\Phi$ on $\dot{Z}$ and $\dot{N}$, or of the incremental functional $\Phi_{n}$ on $Z_{n+1}$ and $N_{n+1}$, does not involve their gradients. Therefore, the minimization with respect to $\dot{N}$ and $\dot{Z}$ in the rate problem, and with respect to $N_{n+1}$ and $Z_{n+1}$ in the incremental problem, may be effected pointwise. This is in contrast to the dependence of the rate functional $\Phi$ on $\dot{\varphi}$ and $T$, or of the incremental functional $\Phi_{n}$ on $\boldsymbol{\varphi}_{n+1}$ and $T_{n+1}$, which involves their gradients. Thus, it follows that the reduced incremental functional

$$
\Phi_{n}\left[\boldsymbol{\varphi}_{n+1}, T_{n+1}\right]=\inf _{N_{n+1}, Z_{n+1}} \Phi_{n}\left[\boldsymbol{\varphi}_{n+1}, T_{n+1}, N_{n+1}, \boldsymbol{Z}_{n+1}\right]
$$

is of the form

$$
\begin{aligned}
\Phi_{n}\left[\boldsymbol{\varphi}_{n+1}, T_{n+1}\right]= & \int_{B} \phi_{n}\left(\boldsymbol{F}_{n+1}, T_{n+1}, \boldsymbol{G}_{n+1}\right) \mathrm{d} V \\
& -\int_{B} R \boldsymbol{B}_{n+1} \cdot\left(\boldsymbol{\varphi}_{n+1}-\boldsymbol{\varphi}_{n}\right) \mathrm{d} V-\int_{\partial_{N} B} \overline{\boldsymbol{T}}_{n+1} \cdot\left(\boldsymbol{\varphi}_{n+1}-\boldsymbol{\varphi}_{n}\right) \mathrm{d} S \\
& +\int_{B} \Delta t R Q_{n+1} \log \frac{T_{n+1}}{T_{n}} \mathrm{~d} V-\int_{\partial_{T} B} \Delta t \bar{H}_{n+1} \log \frac{T_{n+1}}{T_{n}} \mathrm{~d} S
\end{aligned}
$$

where

$$
\phi_{n}\left(\boldsymbol{F}_{n+1}, T_{n+1}, \boldsymbol{G}_{n+1}\right)=\inf _{N_{n+1}, \boldsymbol{Z}_{n+1}}\left\{\left(E_{n+1}-E_{n}\right)-T_{n+1}\left(N_{n+1}-N_{n}\right)+\Delta t \Delta_{n+1}\right\}
$$

may be regarded as a non-equilibrium thermoelastic energy density. The reduced minimum problem is

$$
\inf _{\boldsymbol{\varphi}_{n+1}} \sup _{T_{n+1}} \Phi_{n}\left[\boldsymbol{\varphi}_{n+1}, T_{n+1}\right] .
$$

For very slow processes, the temperature gradients become negligibly small and $\phi_{n}$ reduces to the incremental strain-energy density proposed by Ortiz and Stainier (1999).

\section{Validation: conversion of plastic work to heat}

The study on heating from plastic power may be traced back to the pioneering work of Taylor and Quinney, who performed their seminal experimental work in 1937 (Taylor and Quinney, 1937). In previous theoretical and numerical analyses, it has often been assumed that the local rate of heating is a constant fraction of the plastic power (e.g., Simo and Miehe, 1992; Camacho and Ortiz, 1997; Marusich and Ortiz, 1995). Several experimental studies have been concerned with the determination of the fraction of plastic work converted to heat and the evolution of that ratio with plastic deformation, either by 
calorimetric or infrared imaging techniques (e.g., Chrysochoos et al., 1989; Chrysochoos and Belmahjoub, 1992). Mason et al. (1994) measured temperature in fully dynamic experiments performed over a wide range of strains and strain rates. Hodowany et al. (2000) improved the resolution of thermal detectors and the quality of the measurements by using a high-speed $\mathrm{HgCdTe}$ photoconductive technique. For engineering materials, these experimental results show considerable variation of the heat to plastic-work ratio with both strain and strain rate. Rosakis et al. (2000) developed expressions for the relationship between temperature rise and plastic power based on an internal variable model (see also Chaboche, 1993).

In contrast, within the variational framework the rate of conversion of plastic work into heat is an outcome of the theory and need not be modelled independently. In this section we assess the validity of this aspect of the theory for two different materials: $\alpha$-titanium and A12024-T3. In particular, we calculate temperature histories under the conditions of the Kolsky (split-Hopkinson) pressure bar experiment and compare the predictions with the measurements of Hodowany et al. (2000).

\subsection{The variational form of the heat equation}

We begin by taking a closer look at the form of the heat equation that follows from the variational framework. Using the identity

$$
\dot{N}=-\partial_{T} \dot{A}=-\partial_{T} \boldsymbol{P}^{\mathrm{e}} \cdot \dot{\boldsymbol{F}}-\partial_{T T} A \dot{T}+\partial_{T} \boldsymbol{Y} \cdot \dot{\boldsymbol{Z}},
$$

and introducing the heat capacity per unit undeformed volume at constant deformation

$$
C_{\mathrm{v}}=-T \partial_{T T} A,
$$

energy (2.13) may be recast as

$$
C_{\mathrm{v}} \dot{T}=\boldsymbol{Y} \cdot \dot{\boldsymbol{Z}}+\boldsymbol{P}^{\mathrm{v}} \cdot \dot{\boldsymbol{F}}+T \partial_{T} \boldsymbol{P}^{\mathrm{e}} \cdot \dot{\boldsymbol{F}}-T \partial_{T} \boldsymbol{Y} \cdot \dot{\boldsymbol{Z}}+R Q-\operatorname{Div} \boldsymbol{H} .
$$

We see from this equation that several factors contribute to raising the temperature of the body: the dissipation $\boldsymbol{Y} \cdot \dot{\boldsymbol{Z}}$ due to internal processes; the viscous dissipation $\boldsymbol{P}^{\mathrm{v}} \cdot \dot{\boldsymbol{F}}$; thermoelastic effects accounted for by the term $T \partial_{T} \boldsymbol{P}^{\mathbf{e}} \cdot \dot{\boldsymbol{F}}$; thermal softening accounted for by the term $-T \partial_{T} \boldsymbol{Y} \cdot \dot{\boldsymbol{Z}}$; and the external heat sources and heat conduction.

Eq. (5.3) should be carefully contrasted with a conventional form of the thermomechanically-coupled heat equation widely used in metal plasticity (cf., e.g., Simo and Miehe, 1992; Camacho and Ortiz, 1997; Marusich and Ortiz, 1995), namely,

$$
C_{\mathrm{v}} \dot{T}=\beta\left(\boldsymbol{F}^{\mathrm{eT}} \boldsymbol{P}\right) \cdot \dot{\boldsymbol{F}}^{\mathrm{p}}+R Q-\operatorname{Div} \boldsymbol{H}
$$

where the multiplicative decomposition (2.16) is assumed to be in force for definiteness, and where thermoelastic effects are considered negligible. In this equation $\left(\boldsymbol{F}^{\mathrm{eT}} \boldsymbol{P}\right) \cdot \dot{\boldsymbol{F}}^{\mathrm{p}}$ is the plastic power, which reduces to $\sigma_{i j} \dot{\mathrm{e}}_{i j}^{\mathrm{p}}$ for small strains, and $\beta$ is a factor, sometimes referred to as the Taylor-Quinney factor (Taylor and Quinney, 1937), that measures the fraction of the plastic power that is converted to heat. The conventional practice has been to regard $\beta$ as an additional state function and to model it independently of the remaining state functions. In particular, the model consisting of assuming $\beta$ to be constant has been widely used in engineering practice.

A comparison of forms (5.3) and (5.4) of the heat equation warrants several interesting conclusions. Thus, it is clear that form (5.4) of the heat equation is not variational in 
general. In particular, the linearization of (5.4) is not symmetric in general. Conversely, within the thermodynamic framework the rate of heating is determined explicitly by the constitutive equations and, therefore, need not - and cannot—-be modelled independently.

\subsection{Comparison with experiment}

We begin by specializing the general formulation to the conditions of the Kolsky (splitHopkinson) pressure bar experiment of Hodowany et al. (2000). For simplicity, we assume linearized kinematics and approximate stresses and deformations as one-dimensional. Under these conditions, the strain has the additive decomposition

$$
\varepsilon=\varepsilon^{\mathrm{e}}+\varepsilon^{\mathrm{p}}
$$

where $\varepsilon^{\mathrm{e}}$ and $\varepsilon^{\mathrm{p}}$ are the elastic and plastic part of the strain. We further assume that $\varepsilon^{\mathrm{p}}$ is the sole internal variable. The free energy density is assumed to have the simple form

$$
A\left(\varepsilon, \theta, \varepsilon^{\mathrm{p}}\right)=\frac{C}{2}\left[\varepsilon^{\mathrm{e}}-\alpha\left(\theta-\theta_{0}\right)\right]^{2}+c_{\mathrm{v}} \theta\left(1-\log \frac{\theta}{\theta_{0}}\right)-c_{\mathrm{v}} \theta_{0},
$$

where $C$ is the elastic modulus, $c_{\mathrm{v}}$ is the specific heat at constant volume, $\alpha$ is the thermal expansion coefficient, $\theta$ is the absolute temperature and $\theta_{0}$ is the reference temperature. Equilibrium relation (2.15a) gives the entropy as

$$
\eta=c_{\mathrm{v}} \log \frac{\theta}{\theta_{0}}+\alpha C\left[\varepsilon^{\mathrm{e}}-\alpha\left(\theta-\theta_{0}\right)\right]
$$

Furthermore, we assume a dual kinetic potential of the form

$$
\psi^{*}\left(\dot{\varepsilon}^{\mathrm{p}}, \theta\right)=\frac{m}{m+1} \sigma_{\mathrm{y}}(\theta) \dot{\varepsilon}_{0}^{\mathrm{p}}\left(1+\frac{\dot{\varepsilon}^{\mathrm{p}}}{\dot{\varepsilon}_{0}^{\mathrm{p}}}\right)^{(m+1) / m}+\left(\sigma_{0}\left(\varepsilon^{\mathrm{p}}, \theta\right)-\sigma_{\mathrm{y}}(\theta)\right) \dot{\varepsilon}^{\mathrm{p}}
$$

where $\sigma_{\mathrm{y}}(\theta)$ is the yield stress, $\dot{\varepsilon}_{0}^{\mathrm{p}}$ is a reference plastic strain rate, $m$ is the rate hardening exponent,

$$
\sigma_{0}\left(\varepsilon^{\mathrm{p}}, \theta\right)=\sigma_{\mathrm{y}}(\theta)\left(1+\frac{\varepsilon^{\mathrm{p}}}{\varepsilon_{0}^{\mathrm{p}}}\right)^{1 / n}
$$

is the flow stress, $\varepsilon_{0}^{\mathrm{p}}$ is a reference plastic strain and $n$ is the hardening exponent. Furthermore, we assume a linear thermal softening of the form

$$
\sigma_{\mathrm{y}}(\theta)=\sigma_{\mathrm{y}}\left(\theta_{0}\right)\left[1-\omega\left(\theta-\theta_{0}\right)\right]
$$

Table 1

Material constants for $\alpha$-titanium (Matthew, 2000) and Al2024-T3

\begin{tabular}{llllllllll}
\hline Material & $C(\mathrm{GPa})$ & $\begin{array}{l}\sigma_{\mathrm{y}}\left(\theta_{0}\right) \\
(\mathrm{MPa})\end{array}$ & $\begin{array}{l}c_{\mathrm{v}}\left(\times 10^{6}\right) \\
\left(\mathrm{Jm}^{-3}\right)\end{array}$ & $\varepsilon_{0}^{\mathrm{p}}$ & $\dot{\varepsilon}_{0}^{\mathrm{p}}$ & $n$ & $m$ & $\omega\left(\mathrm{K}^{-1}\right)$ & $\begin{array}{l}\alpha\left(\times 10^{-6}\right) \\
\left(\mathrm{K}^{-1}\right)\end{array}$ \\
\hline$\alpha$-titanium & 116 & 400 & 2.33 & 0.1 & 0.1 & 1.45 & 35 & 0.0019 \\
Al2024-T3 & 69 & 380 & 2.43 & 0.0015 & 0.5 & 5.8 & 10000 & 0.0007 & 2.0 \\
\hline
\end{tabular}





Fig. 1. (a) Stress-strain curves of $\alpha$-titanium at strain rate of $1 \mathrm{~s}^{-1}$ and $3000 \mathrm{~s}^{-1}$. (b) Adiabatic temperature rise as a function of plastic strain for $\alpha$-titanium at strain rate of $1 \mathrm{~s}^{-1}$ and $3000 \mathrm{~s}^{-1}$. Experimental data from Hodowany et al. (2000).
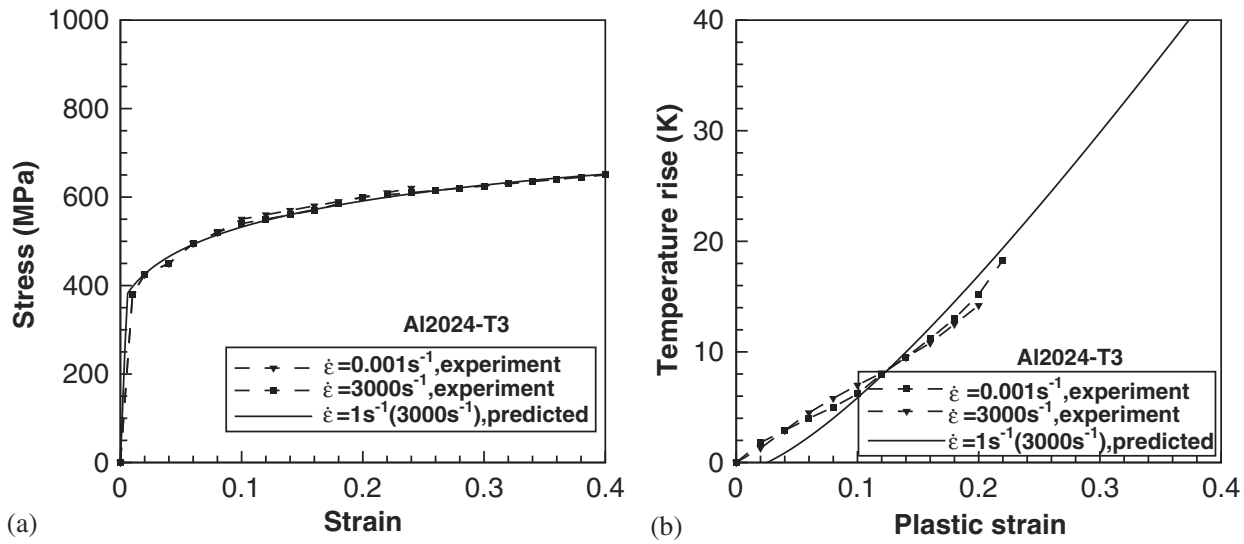

Fig. 2. (a) Stress-strain curves of Al2024-T3 at strain rate $1 \mathrm{~s}^{-1}$ and $3000 \mathrm{~s}^{-1}$. (b) Adiabatic temperature rise as a function of plastic strain for Al2024-T3 at strain rate of $1 \mathrm{~s}^{-1}$ and $3000 \mathrm{~s}^{-1}$. Experimental data from Hodowany et al. (2000).

where $\omega$ is the thermal softening coefficient (cf., e.g., Meyers, 1994). The inverse kinetic relations follow as

$$
y=\frac{\partial \psi^{*}}{\partial \dot{\varepsilon}^{\mathrm{p}}}=\sigma_{\mathrm{y}}(\theta)\left(1+\frac{\dot{\varepsilon}^{\mathrm{p}}}{\dot{\varepsilon}_{0}^{\mathrm{p}}}\right)^{1 / m}+\sigma_{0}\left(\varepsilon^{\mathrm{p}}, \theta\right)-\sigma_{\mathrm{y}}(\theta) .
$$

We apply the preceding model to two materials: rate-dependent $\alpha$-titanium and rateindependent A12024-T3. The material parameters used in the calculations are collected in Table 1. These parameters are fitted to the experimental data of Hodowany et al. (2000). In calculations, we consider two strain rates, $\dot{\varepsilon}=1 \mathrm{~s}^{-1}$ and $\dot{\varepsilon}=3000 \mathrm{~s}^{-1}$. 
The stress-strain curves for $\alpha$-titanium predicted by the model are plotted in Fig. 1a and compared with experiment. It is evident from this figure that the stress-strain curve of $\alpha$ titanium is ostensibly strain-rate sensitive. Fig. $1 \mathrm{~b}$ cross-plots the predicted temperature and plastic strain histories and compares the resulting curves with the experimental data of Hodowany et al. (2000) for strain rates of $1 \mathrm{~s}^{-1}$ and $3000 \mathrm{~s}^{-1}$. Evidently, the experimental and theoretical temperature evolution, including its strain-rate dependence, are in close correspondence. For the lower strain rate case, the slight discrepancy between experimental and numerical temperatures at high strains could be attributed to heat conduction effects not taken into account in the present analysis.

The predicted stress-strain curves and temperature rise for A12024-T3 are plotted in Figs. 2a and b, respectively, and compared with the experimental data of Hodowany et al. (2000). In contrast to $\alpha$-titanium, the stress-strain curve of A12024-T3 and the attendant temperature rise are ostensibly rate-insensitive. Again, the experimental and theoretical temperature evolution, including its strain-rate dependence, are in close correspondence. This agreement suggests that both $\alpha$-titanium and A12024-T3 conform to the variational framework developed in the foregoing, and that the heating rates of these materials need not be modelled independently.

\section{Summary and concluding remarks}

We have developed a variational framework for the coupled thermo-mechanical boundary-value problem for general dissipative solids. In this framework, the equations of motion and the energy balance equation follow jointly as Euler-Lagrange equations of a common potential function. A key element of the present formulation which makes the variational formulation possible is the distinction between an external temperature and an equilibrium temperature. The equilibrium constitutive relations and kinetic relations of the material also follow by taking variations with respect to the internal variables as in previous variational formulations of the isothermal case (Ortiz and Stainier, 1999).

One consequence of the variational formulation of the problem is that the fraction of plastic work converted to heat is predicted by the theory and cannot be modelled independently of the remaining constitutive relations. Therefore, comparison of experimental and theoretical heating rates provides a test of whether a material conforms to the variational framework. The good correspondence between the theoretical predictions and the experimental data of Hodowany et al. (2000) suggests that both $\alpha$-titanium and A12024-T are variational.

A fundamental question that immediately suggests itself is why materials that appear to conform to the variational framework do so. Since the existence of a kinetic potential requires the satisfaction of Onsager-Casimir's reciprocity relations, a closely related question concerns whether such relations follow as a consequence of fundamental principles. The Onsager-Casimir relations do indeed enjoy some grounding in nonequilibrium statistical mechanics and appear to be satisfied by many materials. These considerations notwithstanding, the variational framework furnishes a convenient and powerful tool for modelling general dissipative materials.

\section{Acknowledgments}

We are grateful for support provided by Caltech's NSF/MRSEC Center for the Science and Engineering of Materials, and DARPA's Structural Amorphous Metals program 
through Caltech's Center for Structural Amorphous Metals. We also gratefully acknowledge the support of the Department of Energy through Caltech's ASCI/ASAP Center for Simulating the Dynamic Response of Materials. LS is Research Associate at the Fonds National de la Recherche Scientifique of Belgium.

\section{References}

Ambrosio, L., 2000. Functions of Bounded Variation and Free Discontinuity Problem. Clarendon Press, New York.

Armero, F., Simo, J.C., 1992. A new unconditionally stable fractional step method for nonlinear coupled thermomechanical problems. Int. J. Numer. Methods Eng. 35 (4), 737-766.

Armero, F., Simo, J.C., 1993. A priori stability estimates and unconditionally stable product formula algorithms for nonlinear coupled thermoplasticity. Int. J. Plasticity 9, 749-782.

Aubry, S., Ortiz, M., 2003. The mechanics of deformation-induced subgrain dislocation structures in metallic crystals at large strains. Proc. R. Soc. London A 459, 3131-3158.

Barbu, V., 1976. Nonlinear Semigroups and Differential Equations in Banach Spaces. Noordhoff, Leyden.

Batra, G., 1989. On a principle of virtual work for thermo-elastic bodies. J. Elasticity 21, $131-146$.

Ben-Amoz, M., 1965. On a variational theorem in coupled thermoelasticity. J. Appl. Mech. 32, $943-945$.

Biot, M.A., 1956. Thermoelasticity and irreversible thermodynamics. J. Stat. Phys. 27, 250-253.

Biot, M.A., 1958. Linear thermodynamics and the mechanics of solids. In: Proceedings of the Third US National Congress of Applied Mechanics. ASME, pp. 1-18.

Braides, A., 1998. Approximation of Free-Discontinuity Problems. Springer, New York.

Camacho, G.T., Ortiz, M., 1997. Adaptive Lagrangian modelling of ballistic penetration of metallic targets. Comput. Methods Appl. Mech. Eng. 142, 269-301.

Carstensen, C., Hackl, K., Mielke, A., 2002. Non-convex potentials and microstructures in finite-strain plasticity. Proc. R. Soc. London A 458 (2018), 299-317.

Carter, P., Martin, J.B., 1976. Work bounding functions for plastic materials. J. Appl. Mech. 98, 434-438.

Chaboche, J.L., 1993. Cyclic viscoplastic constitutive equations, part ii: Stored energy-comparison between models and experiments. J. Appl. Mech. 60, 822-828.

Chrysochoos, A., Belmahjoub, F., 1992. Thermographic analysis of thermomechanical couplings. Arch. Mech. 44 (1), 55-68.

Chrysochoos, A., Maisonneuve, O., Martin, G., Caumon, H., Chezeaux, J.C., 1989. Plastic and dissipated work and stored energy. Nucl. Eng. Des. 114, 323-333.

Coleman, B.D., Noll, W., 1963. The thermodynamics of elastic materials with heat conduction and viscosity. Arch. Ration. Mech. Anal. 13 (3), 167-178.

Dal Maso, G., 1993. An Introduction to $\Gamma$-Convergence. Birkhauser, Boston.

Ekelan, I., Temam, R., 1976. Convex analysis and variational problems. Studies in Mathematics and its Applications. North-Holland, Amsterdam.

Han, W., Jensen, S., Reddy, B.D., 1997a. Numerical approximations of problems in plasticity: error analysis and solution algorithms. Numer. Linear Algebra Appl. 4, 191-204.

Han, W., Reddy, B.D., Schroeder, G.C., 1997b. Qualitative and numerical analysis of quasi-static problems in elastoplasticity. SIAM J. Numer. Anal. 34, 143-177

Herrmann, G., 1963. On variational principles in thermoelasticity and heat conduction. Q. Appl. Math. 22, $151-155$.

Hodowany, J., Ravichandran, G., Rosakis, A.J., Rosakis, P., 2000. Partition of plastic work into heat and stored energy in metals. Exp. Mech. 40 (2), 113-123.

Lee, E.H., 1969. Elastic-plastic deformation at finite strains. J. Appl. Mech. 36.

Lubliner, J., 1972. On the thermodynamic foundations of non-linear solid mechanics. Int. J. Non-Linear Mech. 7 , $237-254$

Maier, G., 1969. Some theorems for plastic strain rates and plastic strains. J. Méc. 8, 5.

Marsden, J.E., Hughes, T.J.R., 1983. Mathematical Foundations of Elasticity. Prentice-Hall, Englewood Cliffs, NJ.

Martin, J.B., Ponter, A.R.S., 1966. A note on a work inequality in linear viscoelasticity. Q. Appl. Math. $24,161$.

Marusich, T.D., Ortiz, M., 1995. Modelling and simulation of high-speed machining. Int. J. Numer. Methods Eng. 38, 3675-3694. 
Mason, J.J., Rosakis, A.J., Ravichandran, G., 1994. On the strain and strain-rate dependence of plastic work converted to heat: an experimental study using high-speed infrared detectors and the kolsky bar. Mech. Mater. $17,135-145$.

Matthew, J.D., 2000. Titanium-A Technical Guide. ASM.

Meyers, M.A., 1994. Dynamic Behavior of Materials. Wiley, New York.

Molinari, A., Ortiz, M., 1987. Global viscoelastic behavior of heterogeneous thermoelastic materials. Int. J. Solids Struct. 23 (9), 1285-1300.

Oden, J.T., Reddy, J.N., 1976. Variational Methods in Theoretical Mechanics. Springer, Berlin.

Ortiz, M., Martin, J.B., 1989. Symmetry-preserving return mapping algorithms and incrementally external paths: a unification of concepts. Int. J. Numer. Methods Eng. 28, 1839-1853.

Ortiz, M., Repetto, E.A., Stainier, L., 2000. A theory of subgrain dislocation structures. J. Mech. Phys. Solids 48, 2077-2114.

Ortiz, M., Repetto, E.A., 1999. Nonconvex energy minimization and dislocation structures in ductile single crystals. J. Mech. Phys. Solids 47, 379-462.

Ortiz, M., Stainier, L., 1999. The variational formulation of viscoplastic constitutive updates. Comput. Methods Appl. Mech. Eng. 171 (3-4), 419-444.

Rice, J.R., 1971. Inelastic constitutive relations for solids an internal-variable theory and its applications to metal plasticity. J. Mech. Phys. Solids 19, 433.

Rosakis, P., Rosakis, A.J., Ravichandran, G., Hodowany, J., 2000. A thermodynamic internal variable model for the partition of plastic work into heat and stored energy in metals. J. Mech. Phys. Solids 48 (3), $581-607$.

Rudin, W., 1973. Functional Analysis. McGraw-Hill, New York.

Simo, J.C., Miehe, C., 1992. Associative coupled thermoplasticity at finite strains: formulation, numerical analysis and implementation. Comput. Methods Appl. Mech. Eng. 98, 41-104.

Soechting, J.F., Lance, R.H., 1969. A bounding principle in the theory of work-hardening plasticity. J. Appl. Mech. 36, 228.

Taylor, G.I., Quinney, H., 1937. The latent heat remaining in a metal after cold working. Proc. R. Soc. London A $163,157-181$. 